Case Report

\title{
Gastric Carcinoma Metastases to the Anterior Maxilla: A Rare Case Report
}

\author{
Ayşegul Apaydin, ${ }^{1}$ Ahmet Karadeniz, ${ }^{2}$ Abdullah Yagiz, ${ }^{1}$ Merva Soluk-Tekkesin, ${ }^{3}$ and Sidar Bagbudar ${ }^{4}$ \\ ${ }^{1}$ Department of Oral and Maxillofacial Surgery, Faculty of Dentistry, Istanbul University, Istanbul, 34093 Fatih, Turkey \\ Departments of ${ }^{2}$ Radiation Oncology and ${ }^{4}$ Pathology , Istanbul Faculty of Medicine, Istanbul University, Istanbul, 34093 Fatih, \\ Turkey \\ ${ }^{3}$ Department of Tumor Pathology, Oncology Institute, Istanbul University, Istanbul, 34093 Fatih, Turkey
}

\section{Article History}

Received 5 June 2018

Accepted 19 September 2018
Keywords :

oral metastasis, oral cancer, gastric cancer, maxillary metastasis

\begin{abstract}
It is very rare that metastatic tumors occur in oral region. Gastric carcinoma is typically asymptomatic, it can be discovered in later stage when it metastases.

54 years old male referred to our department with complaint of a mass $(3.5 \times 2.5 \mathrm{~cm})$ which was occurred 2 weeks ago, in the vestibule next to left second incisor.

In the extra-oral examination lip was slightly expanded. No lymphadenopathy was determined in the cervical region. No pathological sign was noticed in the medical history.

In radiological examination has been seen radiolucent lesion with irregular margins.

It was considered as primary malign lesion and resected. Histopathological examination revealed high-grade undifferentiated tumor and the patient was scanned with positron emission tomography (PET). PET showed a mass in stomach, metastasis in the peripheral lymph nodes.

Esophagogastroduodenoscopy performed. It revealed necrotic mass in stomach. Biopsy was made and diagnosed, undifferentiated carcinoma. Chemotherapy was given. His health got worse.

Metastatic tumors originating from gastrointestinal system can spread through Batson's plexus.

When a metastatic tumor occurs as first symptom, it gives an opportunity to cure the disease in early stage. So existence of a malign lesion in oral tissues, it is important to search a possible metastatic relation with any malignancies elsewhere in the body that can lead lethal prognosis.
\end{abstract}

\section{Introduction}

Malignant tumors of the oral region constitute about $5 \%$ of all malignant tumors, of which $1 \%$ are metastatic $(1,2)$. In some cases, oral metastatic tumors are the first indication of an undiagnosed primary tumor(3).

Gastric carcinoma metastasizes of the oral cavity are extremely rare (3). Patients with metastasis in the oral cavity usually develop metastases in other regions of the body (2). In the early period, gastric carcinoma can present with mild or no symptoms. Therefore, the tumor is usually detected in late stages (3). The prognoses of these patients are very poor $(4,5)$.

Correspondence to :

Aysegul Apaydin

E-mail : apaydin@istanbul.edu.tr
Herein, we report a case of gastric carcinoma that emerged as a metastatic lesion in the maxillary gingiva, without any signs, which is rarely reported in the literature.

\section{Case Presentation}

A 54-year-old male patient was admitted to our clinic with the complaint of swelling $(3.5 \times 2.5 \mathrm{~cm})$ of the erythematous surface in the vestibular gingiva of the left maxilla, which he had first noticed 2 weeks previously (Fig. 1). He had no pain, and there was no significant finding in the medical history. He was a heavy smoker.

The intraoral examination revealed poor oral hygiene; there were root caries and decay.

An extra-oral examination revealed that the upper lip was slightly elevated. No lymphadenopathy was detected in the 


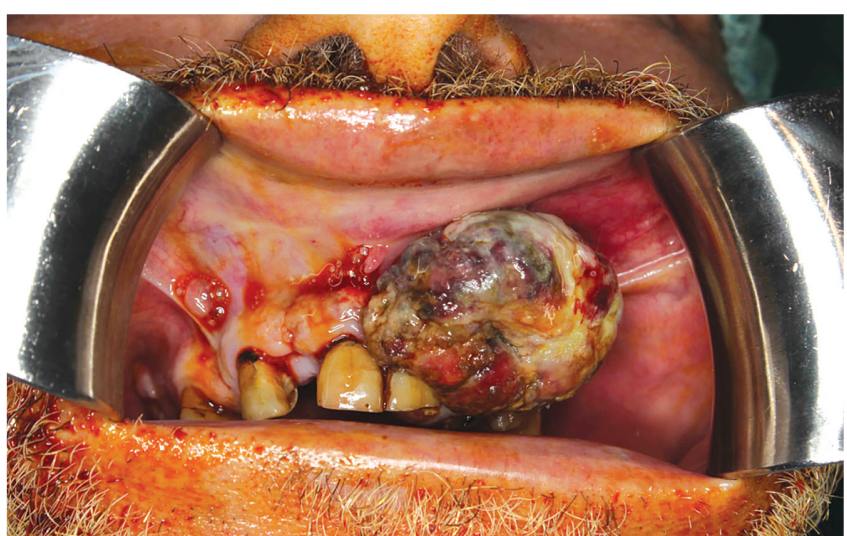

Fig. 1. Pre-operative view of the mass
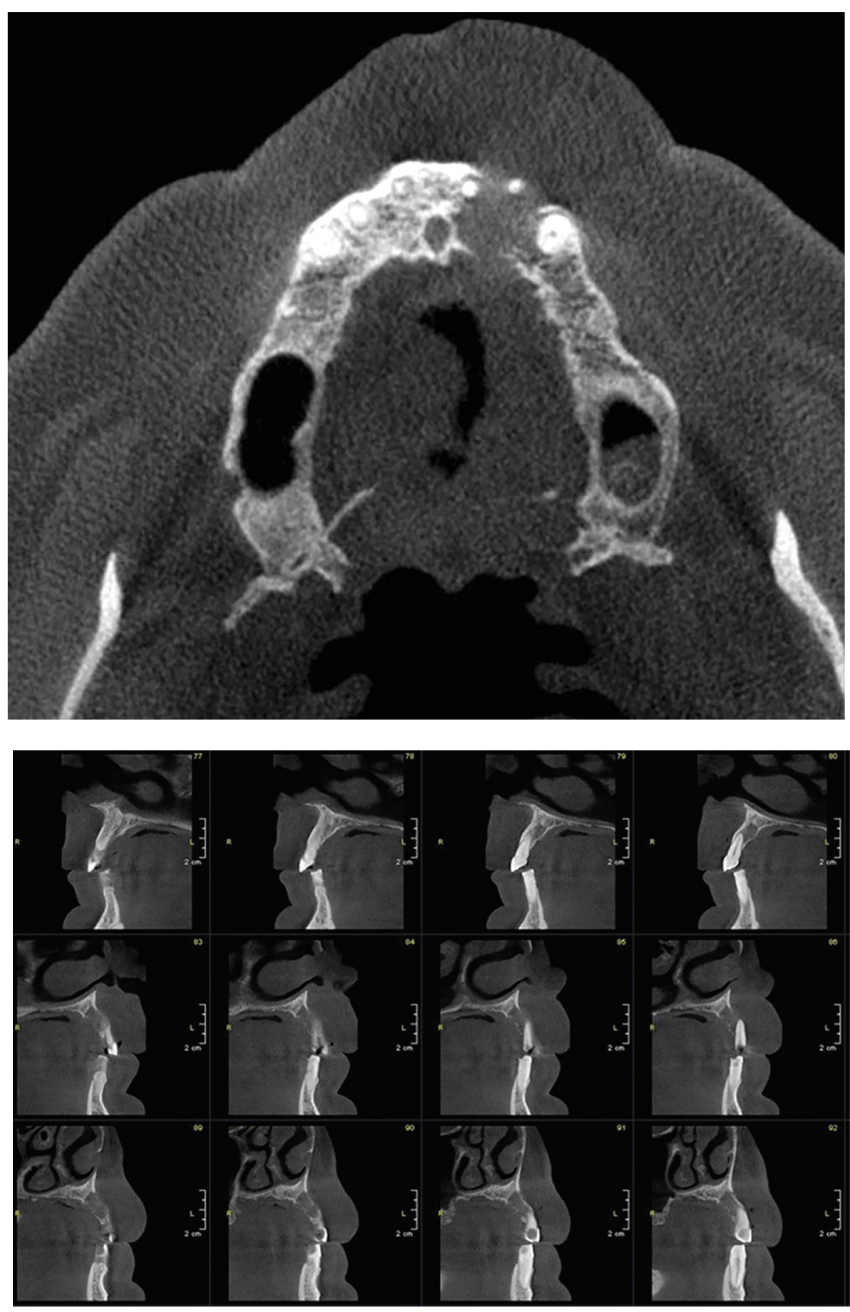

Fig. 2. Cone beam computed tomography view of the osteolytic lesion in maxilla

cervical region. A radiologic examination exposed a radiolucent, osteolytic lesion with irregular margins around the incisal teeth (Fig. 2). The superior edge of the lesion was near the nasal floor, and the margins of the lesion had

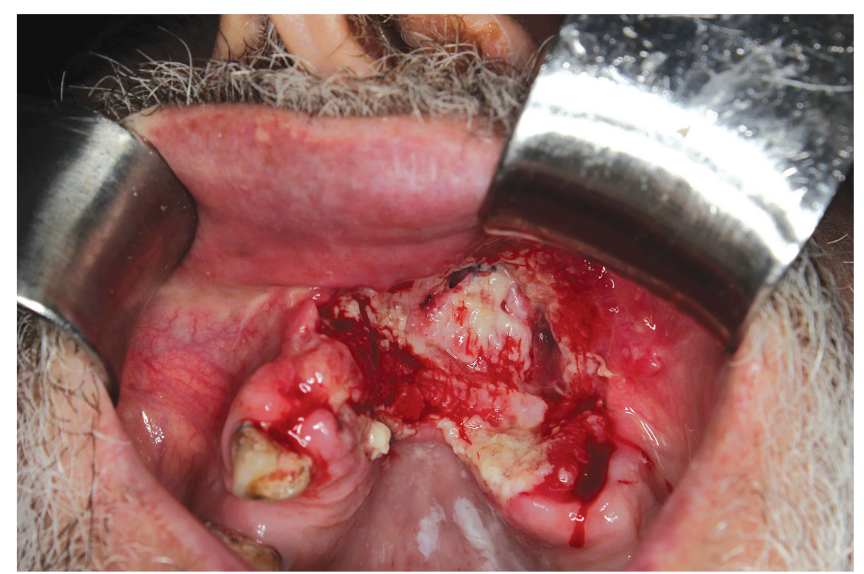

Fig. 3. Post-operative view of the defect

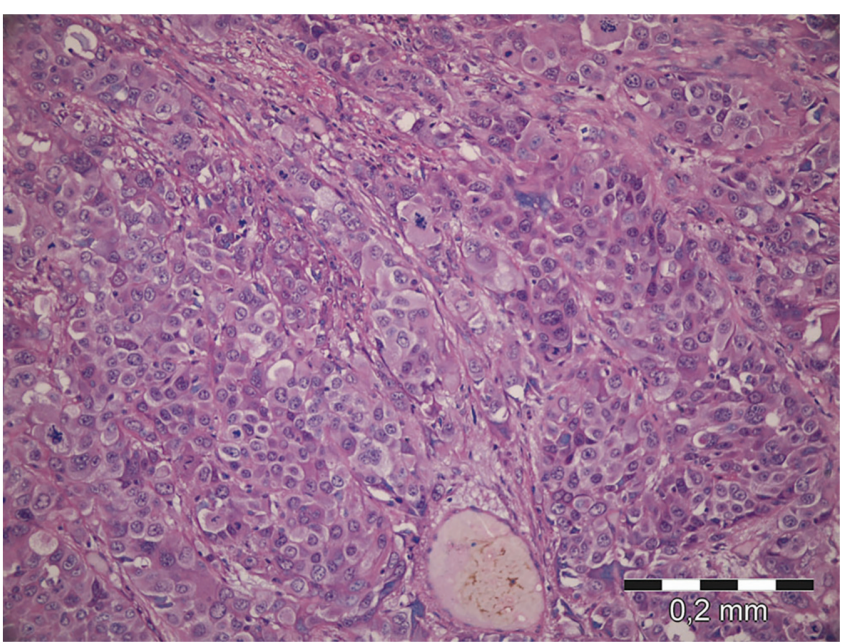

Fig. 4. Photomicrograph showing a solid growth pattern composed of large, polygonal to round tumor cells with distinct nuclei and note that atypical mitoses were also seen, HE X200

merged with the oral and palatal mucosa. The Carcinoembryonic Antigen (CEA) value was $5.83 \mathrm{ng} / \mathrm{mL}$ (reference $<5$ ).

The absence of any other findings and the clinical features of the lesion suggested that it might be a primary malignant tumor. A resection of the lesion was performed (Fig. 3).

Histopathological examination confirmed that the lesion was a high grade undifferentiated malignant tumor (Fig. 4). The tumor had infiltrated to the bone as well as the gingiva.

Although no systemic or clinical symptoms were observed, the patient was referred to the Institute of Oncology because of the possibility of internal organ metastasis.

The patient underwent positron emission tomography (PET). PET imaging with F-18 fluorodeoxyglucose (FDG) showed the involvement of several lymph nodes, the largest 


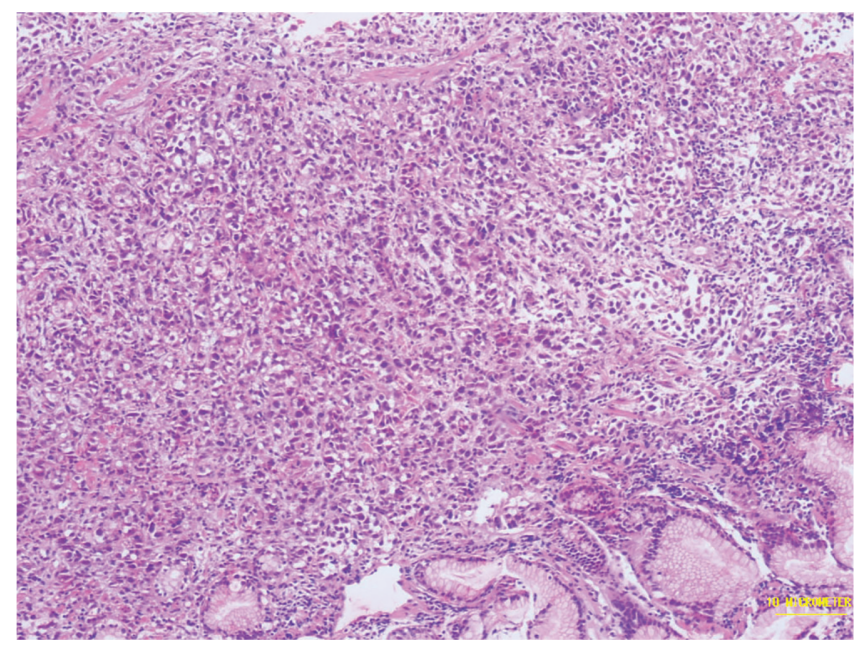

Fig. 5. Photomicrograph of diffuse tumoral infiltration showing pleomorphic and hyperchromatic nuclei, normal gastric foveolar epithelium below, HE X10

of which was $2.8 \mathrm{~cm}$ in left high mediastinum (SUDmax: 6.5). Further details are as follows: $3.6-\mathrm{cm}$ lymph node involvement in the right hilar (SUDmax: 6.0); involvement of $4.5 \times 3 \mathrm{~cm}$ in the right lung (SUDmax: 9,7 ); involvement of $6.4 \times 4.8 \mathrm{~cm}$ in the right adrenal gland (SUDmax: 8.0 ); a mass of $5.8 \times 6.2 \mathrm{~cm}$ between the lesser curvature and celiac in the stomach (SUDmax: 9.7); involvement along a segment of $10 \mathrm{~cm}$ at the level of the gastric antrum (SUDmax: 12.7); involvement of $2.7 \times 2.7 \mathrm{~cm}$ in the tail of the pancreas (SUDmax: 4.8); involvement and mass of 6.4 $\times 4.8 \mathrm{~cm}$ between the intestinal segments at the renal hilus level (SUDmax: 7.3); lymph node involvements in the paraortocaval regions (SUDmax: 2.2); nodular lesion involvements of $3 \mathrm{~cm}$ on the left side at the level of L3, $1 \mathrm{~cm}$ in mesentery, $3.8 \times 1.8 \mathrm{~cm}$ at the level of renal hilus (SUDmax: 2.2 ); and intestinal involvement along a segment of $3.5 \mathrm{~cm}$ in the lower quadrant. The FDG distribution in the neck was determined to be normal.

The patient underwent an esophagogastroduodenoscopy. A mass lesion that surrounded the lumen extending from the proximal gastric corpus to pylorus and of which central mucosa integrity was partly impaired due to necrosis was detected. The pyloric integrity was also impaired. The histopathological diagnosis was ultimately undifferentiated carcinoma (Fig. 5). The patient was prescribed a drug regimen of capecitabine $150 \mathrm{mg}(2 \times 2$ tablet/day) because his overall condition was not good and his ECOG score was 2-3.

Chemotherapy was unfortunately not effective as a

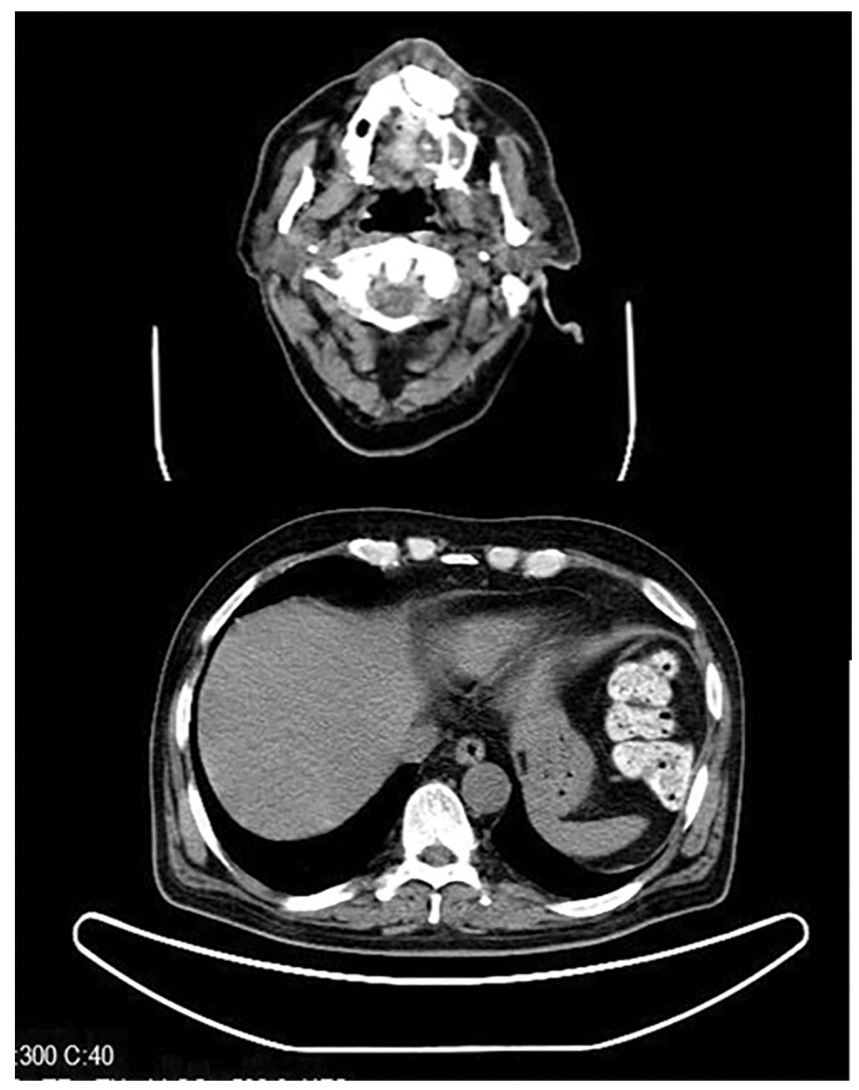

Fig. 6. PET image of the oral and gastric lesion

treatment option. The patient's systemic condition worsened because of liver and multiple progressive intraabdominal metastases, and he died within 5 months.

\section{Discussion}

A small proportion (3.7\%) of metastatic tumors of the oral region originates from gastric carcinoma. The majority of these are adenocarcinomas (40\%) (5).

Close to half (49.2\%) of metastatic tumors occur in hard tissues, i.e., mostly in the mandible(35.6\%), maxilla (7.3\%), and temporal bone (4.5\%). The remaining metastatic tumors $(50.8 \%)$ occur in soft tissues, i. e., mostly in the gingiva (17.7\%), parotid gland (9.8\%), and tongue (6.5\%) (4). In this case report, the lesion covered an equal volume of both the bone and gingiva.

Gingival metastasis is usually seen at advanced stages of tumor development and accompanies other organ metastases. Therefore, the prognosis is poor. Gingival metastatic tumors are differentiated from benign reactive lesions by rapid and progressive growth (6).

Irregularly bordered, radiolucent, and osteolytic lesions 
are seen in $86 \%$ of bone metastases by radiological examination (5). The lesion might shrink if radiotherapy and chemotherapy are effective in osteolytic lesions. In these cases, a radiographically mixed image can be seen due to new bone formation and calcifications (7).

Gastric carcinoma spreads to adjacent organs by direct invasion, peritoneal spread, and lymphatic and hematogenous pathways. Hematogenous spread is the most common mechanism in distant metastasis (3). Head and neck metastases originating from the gastrointestinal tract can reach the region by following Batson's plexus (8). Because our patient had no cervical lymphadenopathy and the FDG distribution in the neck was normal, we believed that metastasis could have occurred via the Batson's plexus. For this reason, the lesion was considered to be primary.

The clinical symptoms of gastric carcinoma include difficulty in digestion, loss of appetite, weight loss, hematemesis, and gastric antral narrowing or pyloric stenosis (3). Our patient had no clinical complaints; therefore, the disease silently metastasized in most of the internal organs, and his gastrointestinal complaints began a month after the oral biopsy.

It has been reported that $20.33 \%$ of patients with a metastatic tumor in the oral region had no primary cancer diagnosis (5). The relationship between primary tumor and metastasis should be considered as a multifaceted and balanced process $(9)$.

\section{Conclusion}

When a malignancy metastasizes in the oral region, the disease is usually advanced. Therefore, the prognosis is poor. Cases in which the metastatic tumor is the first symptom provide an opportunity to discover and treat the primary tumor. For this reason, a detailed systemic examination is important, especially in the presence of a histologically unusual lesion in the oral tissues. Such detailed examinations might help to detect potentially lethal malignancies at an early stage.

\section{Footnotes}

This study was presented as an electronic poster at the International Association of Oral and Maxillofacial Surgeons (IAOMS) -Oral and Maxillofacial Society (ACBID) Joint Congress, 9-13

May, 2018, Antalya, Turkey.

Conflict of Interest: No conflict of interest was declared by the authors.

Financial Disclosure: The authors declared that this study has received no financial support.

\section{References}

1. Carnelio S, Rodrigues G: Maxillary metastasis of gastric adenocarcinoma. Int J Oral-Med Sci, 3: 159-162, 2005.

2. van der Waal RIF, Buter J, van der Waal I: Oral metastases: report of 24 cases. Br J Oral Maxillofac Surg, 41: 3-6, 2003.

3. Umashankar DN, Srinath N, Bavle RM, Bhandari A: Anterior maxillary metastasis of gastric adenocarcinoma: a rare case. J Oral Maxillofac Pathol, 17: 467-470, 2013.

4. McClure SA, Movahed R, Salama A, Ord RA: Maxillofacial metastases: a retrospective review of one institution's 15-year experience. J Oral Maxillofac Surg, 71: 178-188, 2013.

5. Shen M, Kang J, Wen Y, Ying W, Yi J, Hua C, Tang X, Wen Y: Metastatic tumors to the oral and maxillofacial region: a retrospective study of 19 cases in west china and review of the Chinese and English literature. J Oral Maxillofac Surg, 67: 718-737, 2009.

6. Kwon M, Ko S, Cho N, Kim O, Shin H, Baek J, Leem D: Gastric signet-ring cell adenocarcinoma metastatic to the gingiva: a case report. Oral Surg Oral Med Oral Pathol Oral Radiol Endod, 102: 62-66, 2006.

7. Uchiyama Y, Murakami S, Kakimoto N, Nakatani A, Kishino M, Hamab Y, Frukawa S: Diagnostic imaging findings for mandibular metastasis from gastric adenocarcinoma. Oral Surg Oral Med Oral Pathol Oral Radiol Endod, 107: e49-e53, 2009.

8. Batson OV: The function of the vertebral veins and their role in the spread of metastases. Ann Surg, 112: 138-149, 1940.

9. Comen E, Norton L, Massague J: Clinical implications of cancer self-seeding. Nat Rev Clin Oncol, 8: 369-377, 2011. 